\title{
Abnormal Central Venous Oxygen Saturation as a Predictor of Mortality in Patients with Sepsis and Polytrauma in the Surgical Intensive Care Unit
}

\author{
T Vijaya Kumar ${ }^{\oplus 1}$, Srinivas ${ }^{\odot 2}$, A. V. Mahesh ${ }^{\circledR 3}$ \\ ${ }^{1}$ MD, Department of Anesthesiology, Lalitha super speciality Hospital, Guntur, Andhra Pradesh, India, ${ }^{2}$ Consultant Physician and Intensivist, Lalitha super Specialty \\ Hospital, Guntur, Andhra Pradesh, India, ${ }^{3}$ DNB, Department of Anesthesiology, Lalitha Super Speciality Hospital, Guntur, Andhra Pradesh, India.
}

\section{Abstract}

Background: In postoperative heart surgery patients and patients with sepsis, pathological (low and supranormal) central venous oxygen saturation $(\mathrm{ScvO} 2)$ was shown to be associated with lower mortality. The objective is we want to examine the effect of sepsis and polytrauma infection on our surgical ICU and the incidence of morbidity and death in the population. Subjects and Methods: In such patients who met the qualifying criteria and had a history of scepticism and multi-trauma, a retrospective observational review was conducted. We investigated and evaluated the association between SCV02 and mortality and other variables, such as lactate and baseline excess and period of the ICU stay and inotropic assistance within the first 6 hours, using a multivariate model review. Their entry was measured and 6 hours later. Result: The incidence of deaths in the hyperoxia group was 2.4 times higher in comparison to the general population, and the death rate in the hypoxia category was 1.025 times higher. The risk of death for the hyperoxic population was 2.4 times higher. There was no statistically relevant discrepancy of ICU, inotropic treatment, coagulopathy, dialysis, apache-11 score, baseline excess and lactate between the three levels of Scv02. Conclusion: The base excess and Scv02 were not significant when the lactates were calculated to a mortality point, but the $\mathrm{j}$ curve revealed that both the hypoxia group and the hyperoxia mortality had improved in contrast with the normal oxy group. Since the small sample size was sufficient to predict a trend, secondary targets were not appropriate.

Keywords: Central Venous Oxygen Saturation, SCVO2, Normaloxia, Sepsis, Trauma, Lactate.

Corresponding Author: Srinivas, Consultant Physician and Intensivist, Lalitha super Specialty Hospital, Guntur, Andhra Pradesh, India. E-mail: drsrinivasosh@gmail.com

Received: 30 July 2020

Revised: 02 September 2020

Accepted: 12 September 2020

Published: 16 December 2020

\section{Introduction}

In ICU cases, sepsis and trauma are the primary causes of death and illness. Typical properties of the two conditions are systemic inflammation and deformed oxygen supply. Unfortunately, multiple treatments on septic medications have only recently improved their longevity. The three key goals of resuscitation are pre-loads, perfusion and tissue hypoxia.

The Early Goal-Directed Therapy (EGDT) reported improved results in the first 6 hours after admission in hospital in septic patients with tailored haemodynamical parameters. The treatment demonstrated that the oxygen supply was maximised by improved central venous oxygen saturation and increased blood pressure results.

Central venous oxygen saturation occurs by measuring the oxygen flow of the venous blood to the heart. It represents a measure of oxygen use, the relation of oxygen availability and oxygen usage in its critical form. Sepsis and trauma, the oxygenation of the heart's blood, oxygen flow to tissue and oxygen consumption of the various tissues are separate pathways involved.

This disparity can be easily observed and monitored, minimising loss and reducing ICU patients' morbidity and mortality by administering oxygen and utilizing it. ${ }^{[1]}$

A low scvO2 therefore can result in the reduced systemic supply of oxygen, an increase in oxygen production or both. The EGDT protocol addresses poor $\mathrm{ScvO} 2$ by optimising oxygen supply by increasing the oxygen saturations, cardiac efficiency or the capacity to oxygen transfer.

As a consequence, the exchange of oxygen failures can precisely be categorised into three losses: 1) macro-circulatory loss, 2) micro-circulating failure, and 3). Macro-circulatory dysfunction typically is measured by measures such as CVP, 
mean arterial pressure (MAP) and cardiac output. ${ }^{[2]}$

No matter whether the individual's ScvO2 levels are minimal or supranormal. Strong $\mathrm{ScvO} 2$ is a poorly understood alarm indicator for oxygenation of damaged tissue in patients with sepsis. In cardiac surgery patients it has been observed that Supra-normal $\mathrm{ScvO} 2$ levels have traditionally been considered with no therapeutic benefits. ${ }^{[3,4]}$

\section{Subjects and Methods}

Place of Study: Lalitha Super Speciality Hospital Intensive Care Unit (ICU). Guntur.

Sample Size: 80 patients with a diagnosis of sepsis or polytrauma or both.

\section{Inclusion Criteria:}

Patients with a diagnosis of sepsis and polytrauma have been admitted to ICU in the age range of 18 to 80 years.

\section{Exclusion Criteria:}

- Patients with Age $<18$ years or $>80$ years,

- Chronic lung disease

- Pregnant women

- Cardiac disease (moderate to severe left ventricular failure, Intra and extracardiac shunts.

- Liver failure (acute or chronic)

- Central venous catheter patients that do not have the tip in the Superior vena cava.

A blood sample from the central venous catheter, the tip of which is situated in the upper vena cava, was obtained to ensure that the systemic oxygen saturation of the pulse oximeter is greater than 93 per cent as per the unit procedure. At the same moment, an arterial blood sample was collected for examination. For the study six hours after ICU entry, a comparable collection of central venous and arterial samples were taken. A severe score of disease (APACHE II) has been registered 24 hours after hospital admission. Standard treatment was provided to patients. In the datasheet, the need for inotropic medicine, ventilation and renal substitution therapy and their duration is documented during the treatment cycle.

\section{Results}

There were a total of 80 patients who satisfied the requirements for ICU admission. Thirty-three per cent were women and sixty-seven per cent were men. 88 per cent were reported for Septic Diagnosis and 12 per cent were confirmed for Polytrauma.

It indicates that statistically, it is not important for the hyperoxia community to have higher mortality. In the
Table 1: Baseline Characteristics data regarding gender and mortality

\begin{tabular}{|c|c|c|c|c|}
\hline & & \multicolumn{3}{|l|}{ SCVO2 } \\
\hline & & $\begin{array}{l}\text { Hypoxia } \\
(<60)\end{array}$ & $\begin{array}{l}\text { Normoxia } \\
(60 \text { to } \\
75)\end{array}$ & $\begin{array}{l}\text { Hyperoxia } \\
(75.1 \text { to } \\
100)\end{array}$ \\
\hline \multirow[t]{2}{*}{ Gender } & Males & 8 & 28 & 23 \\
\hline & Female & 3 & 10 & 8 \\
\hline \multirow[t]{2}{*}{ Mortality } & Yes & 1 & 2 & 5 \\
\hline & No & 11 & 35 & 27 \\
\hline
\end{tabular}

Table 2: Correlation of $\mathrm{ScvO2}$ to mortality at the time of admission and after 6 hours

\begin{tabular}{|llll|}
\hline SCVO2 & & $\begin{array}{l}\text { No. of } \\
\text { patients } \\
\text { Alive }\end{array}$ & $\begin{array}{l}\text { No. } \\
\text { patients } \\
\text { Dead }\end{array}$ \\
\hline At the time of & Low & 9 & - \\
& Normal & 34 & 1 \\
\hline & High & 24 & 4 \\
\hline 6 Hours & Low & 14 & - \\
\hline & Normal & 34 & 1 \\
\hline & High & 24 & 4 \\
\hline
\end{tabular}

hyperoxy population, death about normoxia was 2.4 times higher, but not statistically significant. In the hyperoxia population, the risk for mortality was 2.4 times higher, but in comparison with the hyperoxia p-value was not significant.

\begin{tabular}{llll|}
\hline \multicolumn{4}{l}{ Table 3: Correlation of oxygen extraction ratio to ScvO2 } \\
\hline Oxygen & SCVO2 & & \\
& Low & Normal & High \\
\hline Low & 4 & 34 & 17 \\
Normal & - & - & 14 \\
High & 7 & 4 & - \\
\hline
\end{tabular}

The oxygen extraction ratio was marginally lower in comparison to that of the hyperoxy group.

The correlation of SCVO2 with ICU stay, dialysis, duration of inotropic treatment and coagulopathy involvement showed that both hypoxia and the population of hyperoxia needed a slightly longer term of inotropic, additional need for dialysis and coagulopathy.

There was no statistically meaningful association between the three SCV02 classes as compared to the APACHE II ranking, base excess, lactate point. 


\begin{tabular}{|c|c|c|}
\hline SCOV2 & Coagulopathy & $\begin{array}{l}\text { No Coagulopa- } \\
\text { thy }\end{array}$ \\
\hline Low & 1 & 2 \\
\hline Normal & 5 & 41 \\
\hline High & 9 & 22 \\
\hline
\end{tabular}

Table 5: ScvO2 and need for dialysis

\begin{tabular}{lll}
\hline SCOV2 & Dialysis & No Dialysis \\
Low & 1 & 10 \\
Normal & 2 & 36 \\
High & 4 & 27 \\
\hline
\end{tabular}

Table 6: cvO2 and lactates

\begin{tabular}{lll}
\hline SCOV2 & High Lactates & Low Lactates \\
Low & 7 & 1 \\
Normal & 19 & 17 \\
High & 15 & 13 \\
\hline
\end{tabular}

Table 7: Correlation of lactate levels to mortality

\begin{tabular}{lll} 
& Alive & Death \\
$\begin{array}{l}\text { Normal Lac- } \\
\text { tate }\end{array}$ & 33 & 1 \\
High Lactate & 39 & 7 \\
\hline
\end{tabular}

\section{Discussion}

A Pope et al. multi-centre study of patients with sepsis was shown to be consistent with decreased mortality in both the hypoxia and the hyperoxia levels of the average SCVO2. A multi-faceted assessment of the same group indicated that hyperoxia was correlated with decreased mortality. There have been a few types of research to confirm this connexion. ${ }^{[1]}$ Multivariate study found a high risk of mortality in patients with hyperoxia (SCVO2 $>75$ percent) on admission, and in patients with normoxia and hypoxia 6 hours after admission, within a large population of SICU-patients. These guidelines show that venous hyperoxia (or insufficient use of oxygen) can be of potential significance in the case of sepsis and polytrauma.

While low SCVO2 may be a marker for macrocirculatory failure, high SCVO2 may be a microcirculatory or mitochondrial failure. In both cases, further studies on the cause of decreased oxygen consumption and alternative solutions must be carried out.

Also, with adequate blood pressure and ample cardiac output, also referred to as the enigmatic shock, Tissue hypoperfusion may be likely. In the case of adequate substrate distribution, the blood flow could be maldistributed at the microvascular level or could be associated with a mitochondrial breakdown.

EGDT is focused on improved systemic availability of oxygen or macrocirculations by raising intravenous preload, raising oxygen delivery capability with red cells, increasing inotropic oxygen flow and an improvement in the pressure of vasoconstrictive perfusion. Microcirculation failure and mitochondrial failure can result in dysoxia, even if the macrocirculatory failure is fixed. ${ }^{[5]}$

In a study by Mikkelsen, ${ }^{[6]}$ initial elevated serum lactate patients in severe sepsis were associated with death irrespective of the clinically obvious shock. While not statistically meaningful, our findings imply greater mortality in the higher level of lactate ( $>2$ mmols). The findings further show that in patients with hyperoxia, the inotropes are slightly longer and the need for coagulopathic dialysis is higher.

While the meantime of ICU in the hyperoxia population was shorter, this was presumably attributed to the early mortality of these patients. Even in the population with hyperoxia, the oxygen consumption ratio was a lower yet higher mortality. It reinforces the concept of cell hypoxia after a major increase in macrocirculatory collapse, leading to microcirculatory and mitochondrial failure. In 27 critically ill septic patients, who had skeletal muscle biopsies within 24 hours of ICU intake, the therapeutic value of mitochondrial dysfunction in septic shock was seen.

In 11 patients who died of sepsis, skeletal muscle ATP concentrations were significantly lower than in 15 survivors, the marker for mitochondrial oxidative phosphorylation (MOPS). Thus, the cytopathic (or histotoxic) anoxia which cannot use the oxygen given may explain cell injury and death from sepsis. The most important cause of sepsis could be microcirculation (i.e. capillary). A reduction in the number of working capillaries due to shunts coincides with sepsis. The microthrombi often associate with a loss of blood supply. ${ }^{[7]}$

Sepsis induces phenotypic variations in endothelial cells. Overt and indirect connexions exist between endothelial cells and components of the bacterial wall. Hyperlactemia in these cases means that the microcirculation blood, even after resuscitation and macrocirculation normalisation, is being not distributed properly. Hypoperfusion is a consequence of microcirculation. Such shutting down of inadequate microcirculation networks in the organ beds promotes blood loss, thus reducing oxygen supply from the arterial to the venous field by hypoxic microcirculation.

Even with a sufficient macrocirculatory flow, microcirculatory failure can prevent the oxygen required and available from reaching the cells in vital organs. ${ }^{[8-10]}$ A further revitalization technique is then required to evaluate microcirculation damage and use microcirculation-enhancing therapy. You should 
consider some causes of mitochondrial dysfunction and treatment methods to address those defects. During adequate global oxygen supply, we have found that high SCVO2 was linked to increased death and that these observations are a target for further studies.

\section{Conclusion}

The base excess and Scv02 were not significant when lactates were calculated to the point of mortality, but a curve in $\mathrm{j}$ form was showing greater mortality both in hypoxia groups and in hyperoxia relative to normalmoxia in the groups. Since the small sample size was sufficient to predict a trend, secondary targets were not met.

\section{References}

1. Pope JV, Jones AE, Gaieski DF, Arnold RC, Trzeciak S, Shapiro NI. Multicenter Study of Central Venous Oxygen Saturation (ScvO2) as a Predictor of Mortality in Patients With Sepsis. Ann Emerg Med. 2010;55(1):40-46.e1. Available from: https://doi.org/10.1016/j.annemergmed.2009.08.014.

2. Perz S, Uhlig T, Kohl M, Bredle DL, Reinhart K, Bauer $\mathrm{M}$, et al. Low and "supranormal" central venous oxygen saturation and markers of tissue hypoxia in cardiac surgery patients: a prospective observational study. Intensive Care Med . 2011;37(1):52-59. Available from: https://doi.org/10.1007/ s00134-010-1980-8.

3. Nebout S, Pirracchio R. Should We Monitor ScVO2 in Critically Ill Patients? Cardiol Res Pract. 2012;2012:370697. Available from: https://dx.doi.org/10.1155/2012/370697.

4. Balzer F, Sander M, Simon M, Spies C, Habicher M, Treskatsch S. High central venous saturation after cardiac surgery is associated with increased organ failure and long-term mortality: an observational cross-sectional study. Crit Care. 2015;19(1):168. Available from: https://dx.doi.org/10.1186/ s13054-015-0889-6.

5. Tyagi A, Sethi AK, Girotra G, Mohta M. The Microcirculation in Sepsis. Indian J Anaesth. 2009;53(3):281-293.

6. Mikkelsen ME, Miltiades AN, Gaieski DF, Goyal M, Fuchs $\mathrm{BD}$, Shah CV, et al. Serum lactate is associated with mortality in severe sepsis independent of organ failure and shock*. Crit Care Med. 2009;37(5):1670-1677. Available from: https://dx. doi.org/10.1097/ccm.0b013e31819fcf68.

7. ;. Available from: http://www.thelancet.com/journals/lancet/ article/PIIS0140-6736(02)09459-X/fulltext?refuid=S01960644.

8. ;2006. Available from: http://www.ncbi.nlm.nih.gov/pubmed/ 16682924.

9. Filippo AD, Gonnelli C, Perretta L, Zagli G, Spina R, Chiostri $\mathrm{M}$, et al. Low central venous saturation predicts poor outcome in patients with brain injury after major trauma: a prospective observational study. Scand J Trauma Resusc Emerg Med. 2009;17(1):23-23. Available from: https://dx.doi.org/10.1186/ 1757-7241-17-23.

10. Bakker J, Schieveld SJM, Brinkert W. Serum lactate level as a indicator of tissue hypoxia in severely ill patients. Ned Tijdschr Geneeskd. 2000;144(16):737-741.

Copyright: (C) the author(s), 2020. It is an open-access article distributed under the terms of the Creative Commons Attribution License (CC BY 4.0), which permits authors to retain ownership of the copyright for their content, and allow anyone to download, reuse, reprint, modify, distribute and/or copy the content as long as the original authors and source are cited.

How to cite this article: Kumar TV, S, Mahesh AV. Abnormal Central Venous Oxygen Saturation as a Predictor of Mortality in Patients with Sepsis and Polytrauma in the Surgical Intensive Care Unit. Acad. Anesthesiol. Int. 2020;5(2):53-56.

DOI: dx.doi.org/10.21276/aan.2020.5.2.11

Source of Support: Nil, Conflict of Interest: None declared. 